Research Article

\title{
Christ-Lee Model: (Anti-)chiral Supervariable Approach to BRST Formalism
}

\author{
B. Chauhan $(D)$ and S. Kumar $(D)$ \\ Physics Department, Centre of Advance Studies, Institute of Science, Banaras Hindu University, Varanasi 221 005, India \\ Correspondence should be addressed to B. Chauhan; bchauhan501@gmail.com
}

Received 9 February 2021; Accepted 13 October 2021; Published 28 October 2021

Academic Editor: Muhammad Farasat Shamir

Copyright (C) 2021 B. Chauhan and S. Kumar. This is an open access article distributed under the Creative Commons Attribution License, which permits unrestricted use, distribution, and reproduction in any medium, provided the original work is properly cited. The publication of this article was funded by SCOAP 3 .

We derive the off-shell nilpotent of order two and absolutely anticommuting Becchi-Rouet-Stora-Tyutin (BRST), anti-BRST, and (anti-)co-BRST symmetry transformations for the Christ-Lee (CL) model in one $(0+1)$-dimension (1D) of spacetime by exploiting the (anti-)chiral supervariable approach (ACSA) to BRST formalism where the quantum symmetry (i.e., (anti-)BRST along with (anti-)co-BRST) invariant quantities play a crucial role. We prove the nilpotency and absolute anticommutativity properties of the (anti-)BRST along with (anti-)co-BRST conserved charges within the scope of ACSA to BRST formalism where we take only one Grassmannian variable into account. We also show the (anti-)BRST and (anti-)co-BRST invariances of the Lagrangian within the scope of ACSA.

\section{Introduction}

Gauge theories describe three (i.e., strong, weak, and electromagnetic) out of four fundamental interactions of nature which are characterized by first-class constraints in the context of Dirac's prescription for the classification scheme of constraints $[1,2]$. The existence of the first-class constraints, in a given system, is the key signature of a gauge theory. Many interesting theories, in the domain of physics, are expressed by the suitable Lagrangians that are invariant under the gauge symmetry transformations. These symmetries are generated by the first-class constraints in a given gauge theory. For the covariant canonical quantization of the gauge theory, the Becchi-Rouet-Stora-Tyutin (BRST) quantization procedure plays a decisive role where we replace the infinitesimal local gauge parameter by ghost and antighost fields [3-6]. Thus, in this formalism, we have two fermionic-type global BRST $\left(s_{b}\right)$ and anti-BRST $\left(s_{a b}\right)$ transformations at the quantum level (for a given local gauge symmetry transformation at the classical level). These symmetry transformations are endowed with two important properties: (i) nilpotency of order two (i.e., $s_{b}^{2}=0, s_{a b}^{2}=0$ ) and (ii) absolute anticommutativity (i.e., $s_{b} s_{a b}+s_{a b} s_{b}=0$ ).
The first property signifies that these quantum BRST and anti-BRST symmetry transformations are fermionic in nature whereas the second property shows that both symmetry transformations are linearly independent of each other. Besides the (anti-)BRST symmetry transformations, we have two more fermionic and linearly independent symmetry transformations which are christened as the co-BRST $\left(s_{d}\right)$ and anti-co-BRST $\left(s_{a d}\right)$ symmetry transformations. The latter fermionic-type symmetry transformations are valid for any $D$-dimensional $p$-form $(p=1,2,3, \cdots)$ gauge theories in $D=2 p$ dimensions of spacetime. We point out that there are some specific systems such as rigid rotor and ChristLee (CL) model in one dimension (1D) that respect the (anti-)co-BRST transformations along with the (anti-)BRST transformations [7-10].

The geometrical interpretation and the emergence of nilpotent (anti-)BRST symmetry transformations have been shown within the ambit of Bonora-Tonin (BT) superfield formalism [11-13] where the Grassmannian variables $(\vartheta, \bar{\vartheta}$ ) and their corresponding derivatives $\left(\partial_{\vartheta}, \partial_{\bar{\vartheta}}\right)$ (with properties $\vartheta^{2}=\bar{\vartheta}^{2}=0, \partial_{\vartheta}^{2}=\partial_{\bar{\vartheta}}^{2}=0$ and $\vartheta \bar{\vartheta}+\bar{\vartheta} \vartheta=0, \partial_{\vartheta} \partial_{\bar{\vartheta}}+\partial_{\bar{\vartheta}} \partial_{\vartheta}=$ $0)$ play a very important role. In BT-superfield approach, we see the connections between the (anti-)BRST symmetry 
transformations $\left(s_{(a) b}\right)$ (with properties $s_{b}^{2}=s_{a b}^{2}=0$ and $s_{b}$ $\left.s_{a b}+s_{a b} s_{b}=0\right)$ and Grassmannian translational generators $\left(\partial_{\vartheta}, \partial_{\bar{\vartheta}}\right)$ because of the fact that both have the same algebraic structure. In this formalism, any $D$-dimensional Minkowskian manifold is generalized onto the $(D, 2)$-dimensional supermanifold. This suitably chosen supermanifold is denoted by the superspace coordinates $\left(x^{\mu}, \vartheta, \bar{\vartheta}\right)$ where $x^{\mu}($ $\mu=0,1,2, \cdots, D-1)$ are the spacetime coordinates and $(\vartheta$, $\bar{\vartheta})$ are a pair of Grassmannian variables.

The CL model is one of the simplest examples of gaugeinvariant system which is described by a singular Lagrangian [14]. Physically, the CL model represents the motion of a point particle moving in a plane under the influence of a central potential. The CL model has been studied at the classical and quantum levels in different perspectives [14-20]. This model is endowed with the first-class constraints in the Dirac's terminology for the classification scheme of constraints $[1,2]$. This model is also quantized by using the Faddeev-Jackiw quantization where all the primary and derived constraints are treated on equal footing without any type of further classification [20]. Within the framework of BRST formalism, the CL model respects six independent continuous symmetries (i.e., BRST, anti-BRST, co-BRST, anti-co-BRST, ghost-scale, and bosonic symmetries) (see, e.g., [9] for detail). The BT-superfield formalism has been applied to obtain the absolutely anticommuting and offshell nilpotent (anti-)BRST as well as (anti-)co-BRST symmetry transformations where the techniques of celebrated horizontality condition (HC) and dual horizontality condition have been used [10], respectively.

In our recent set of papers [21-25], we have used a newly proposed formalism which has been called by us as the (anti)chiral superfield/supervariable approach (ACSA) to BRST formalism. In this approach, we take into account only one Grassmannian variable in the expression for the superfield/supervariable. Thus, the resulting superfield/supervariable turns into (anti-)chiral version of the superfield/supervariable. In other words, in this formalism, any $D$-dimensional Minkowskian manifold is generalized onto $(D, 1)$-dimensional super-submanifolds of the most general $(D, 2)$ -dimensional supermanifold. The proof of the absolute anticommutativity property of Noether's conserved charges is obvious in the case of BT-superfield formalism where the full super expansions of superfields/supervariables are taken into account. In the case of ACSA, we have also been able to show the nilpotency and absolute anticommutativity properties of conserved charges despite the fact that we have taken only one Grassmannian variable into account. In our present endeavor, we derive the (anti-)BRST together with (anti-)co-BRST symmetry transformations where some specific sets of (anti-)BRST and (anti-)co-BRST invariant restrictions play a very important role. We also show the absolute anticommutativity as well as nilpotency properties of (anti-)BRST and (anti-)co-BRST conserved charges within the realm of ACSA to BRST formalism.

Against the background of the above paragraph, it has been found that the nilpotency of the $\mathcal{N}=2$ super charges is true for any $\mathcal{N}=2$ supersymmetric (SUSY) quantum mechanical models (see, e.g., [26-28]) within the ambit of (anti-)chiral supervariable approach to BRST formalism. However, the application of ACSA, in the realm of $\mathcal{N}=2$ SUSY quantum mechanical model, does not lead to the absolute anticommutativity of the $\mathcal{N}=2$ super conserved charges. Rather, it has been found that the anticommutator of the above $\mathcal{N}=2$ SUSY conserved charges leads to the time translation of the variable on which it acts. Thus, it is crystal clear that ACSA to BRST formalism does not lead to the derivation of absolute anticommutativity of the charges for all $\mathcal{N}=2$ SUSY theories.

The different sections of our present paper are arranged as follows. In Section 2, we discuss the (anti-)BRST and (anti-)coBRST symmetry transformations for the CL model and derive the conserved charges. Our Section 3 deals with the ACSA to BRST formalism where we derive the (anti-)BRST symmetry transformations. Section 4 is devoted to the derivation of (anti-)co-BRST symmetry transformations by using the ACSA to BRST formalism where the super expansions of (anti-)chiral supervariables are utilized in a fruitful manner. In Section 5, we express the conserved (anti-)BRST and (anti-)co-BRST charges on the $(1,1)$-dimensional super-submanifolds (of the most general $(1,2)$-dimensional supermanifold) on which our theory is generalized and provide the proof of nilpotency and absolute anticommutativity properties of the (anti-)BRST along with (anti-)co-BRST charges within the ambit of ACSA to BRST formalism. In Section 6, we discuss the (anti-)BRST and (anti-)co-BRST invariances of the Lagrangian within the scope of ACSA. Finally, we point out our key results and discovery in Section 7 and mention a few future scopes for further investigation.

\section{Preliminaries: Symmetries and Their Corresponding Generator for the Christ- Lee Model}

The first-order and gauge-invariant Lagrangian of the Christ-Lee (CL) model [the other equivalent second-order Lagrangian $\left(L_{s}\right)$ [14] associated with the Christ-Lee model is $L_{s}=(1 / 2) \dot{r}^{2}+(1 / 2) \dot{r}^{2}(\dot{\varphi}-z)^{2}-V(r)$; but we choose only the first-order Lagrangian $L_{f}$ in our present work because it respects maximum symmetry transformations] in $(0+1)$ -dimension (1D) of spacetime in polar coordinates system is given by $[14,16,19]$

$$
L_{f}=\dot{r} p_{r}+\dot{\varphi} p_{\varphi}-\frac{1}{2} p_{r}^{2}-\frac{1}{2 r^{2}} p_{\varphi}^{2}-z p_{\varphi}-V(r)
$$

where $\dot{r}$ and $\dot{\varphi}$ are the generalized velocities, $p_{r}$ and $p_{\varphi}$ are their corresponding canonical momenta, respectively, and $z$ is a Lagrange multiplier which enforces a constraint $p_{\varphi} \approx 0$. This Lagrangian explains that a two-dimensional particle moves under the influence of the central potential $V(r)$ bounded from below. 
The above system has a primary constraint as follows:

$$
\Phi_{1}=\frac{\partial L_{f}}{\partial \dot{z}}=p_{z} \approx 0
$$

The time derivative of the primary constraint $\Phi_{1}$ leads to the following secondary constraint:

$$
\frac{d \Phi_{1}}{d \tau}=\frac{d}{d \tau}\left(\frac{\partial L_{f}}{\partial \dot{z}}\right) \approx 0 \Rightarrow \Phi_{2}=p_{\varphi} \approx 0
$$

It is clear that both $\Phi_{1}$ and $\Phi_{2}$ are first-class constraints. The gauge symmetry transformation generator can be written in terms of first-class constraints as

$$
G=\dot{\chi}(\tau) \Phi_{1}+\chi(\tau) \Phi_{2}
$$

where $\chi(\tau)$ is an infinitesimal and time-dependent local gauge parameter and $\dot{\chi}(\tau)=d \chi / d \tau$. Using the definition of a generator

$$
\delta \phi(\tau)=-i[\phi(\tau), G], \quad \phi=r, p_{r}, \varphi, p_{\varphi}, z
$$

where $\phi$ is the generic variable that is present in the firstorder Lagrangian $L_{f}$. We deduce the following local gauge transformations by exploiting Equation (5), namely,

$$
\begin{gathered}
\delta z(\tau)=\dot{\chi}(\tau), \\
\delta \varphi(\tau)=\chi(\tau), \\
\delta\left[r(\tau), p_{r}(\tau), p_{\varphi}(\tau), V(r)\right]=0 .
\end{gathered}
$$

It is elementary to check that, under the above local gauge symmetry transformations, the Lagrangian under consideration remains invariant (i.e., $\delta L_{f}=0$ ).

The (anti-)BRST invariant Lagrangian for the $(0+1)$ -dimensional CL model containing the gauge-fixing and Faddeev-Popov ghost terms is given by [15]

$$
\begin{aligned}
L= & \dot{r} p_{r}+\dot{\varphi} p_{\varphi}-\frac{1}{2} p_{r}^{2}-\frac{1}{2 r^{2}} p_{\varphi}^{2}-z p_{\varphi}-V(r) \\
& +\frac{1}{2} \mathscr{B}^{2}+\mathscr{B}(\dot{z}+\varphi)+i \bar{C} C-i \dot{\bar{C}} \dot{C}
\end{aligned}
$$

where the Nakanishi-Lautrup-type auxiliary variable $\mathscr{B}$ is used to linearize the gauge-fixing term and the FaddeevPopov (anti-)ghost variables $(\bar{C}) C$ are used to make the Lagrangian BRST invariant. These fermionic variables $(\bar{C})$ $C$ (with $\left.C^{2}=\bar{C}^{2}=0, C \bar{C}+\bar{C} C=0\right)$ have ghost numbers $(-1$ )+1 , respectively. The above Lagrangian respects the following off-shell nilpotent (i.e., $s_{(a) b}^{2}=s_{(a) d}^{2}=0$ ) and absolutely anticommuting (i.e., $s_{b} s_{a b}+s_{a b} s_{b}=0$ and $s_{d} s_{a d}+s_{a d} s_{d}=0$ )
(anti-)BRST along with (anti-)co-BRST symmetry transformations:

$$
\begin{aligned}
s_{a b} z & =\dot{\bar{C}}, \\
s_{a b} z & =\dot{\bar{C}}, \\
s_{a b} \varphi & =\bar{C}, \\
s_{a b} C & =-i \mathscr{B}, \\
s_{a b}\left[r, p_{r}, p_{\varphi}, \mathscr{B}, \bar{C}\right] & =0, \\
s_{b} z & =\dot{C}, \\
s_{b} \varphi & =C, \\
s_{b} \bar{C} & =i \mathscr{B}, \\
s_{b}\left[r, p_{r}, p_{\varphi}, \mathscr{B}, C\right] & =0, \\
s_{d}\left[r, p_{r}, p_{\varphi}, \mathscr{B}, \bar{C}\right] & =0 . \\
s_{d} C & =i p_{\varphi}, \\
s_{a d} z & =C, \\
s_{a d} \varphi & =-\dot{C}, \\
s_{a d} \bar{C} & =-i p_{\varphi}, \\
s_{d} z & =\bar{C}, \\
\mathscr{B}, p_{r}, p_{\varphi}, C & =0, \\
s_{d}, &
\end{aligned}
$$

It can be clearly checked that under the above (anti)BRST (Equation (8)) and (anti-)co-BRST (Equation (9)) symmetry transformations the Lagrangian (Equation (7)) remains quasi-invariant (i.e., modulo a total time derivative):

$$
\begin{gathered}
s_{b} L=\frac{d}{d \tau}(\mathscr{B} \dot{C}), \\
s_{a b} L=\frac{d}{d \tau}(\mathscr{B} \dot{\bar{C}}), \\
s_{d} L=-\frac{d}{d \tau}\left(p_{\varphi} \dot{\bar{C}}\right), \\
s_{a d} L=-\frac{d}{d \tau}\left(p_{\varphi} \dot{C}\right) .
\end{gathered}
$$

As a result, the action integral $S=\int d \tau L$ remains invariant under the (anti-)BRST as well as (anti-)co-BRST symmetry transformations (i.e., $s_{(a) b} S=0, s_{(a) d} S=0$ ). According to Noether's theorem, the invariance of the above Lagrangian under the nilpotent (anti-)BRST together with (anti-)coBRST symmetry transformations leads to the following 
(anti-)BRST charges $\left[Q_{(a) b}\right]$ and (anti-)co-BRST charges [ $\left.Q_{(a) d}\right]$, namely,

$$
\begin{aligned}
& Q_{a b}=\mathscr{B} \dot{\bar{C}}+p_{\varphi} \bar{C} \equiv \mathscr{B} \dot{\bar{C}}-\mathscr{B} \bar{C}, \\
& Q_{b}=\mathscr{B} \dot{C}+p_{\varphi} C \equiv \mathscr{B} \dot{C}-\mathscr{B} C, \\
& Q_{a d}=\mathscr{B} C-p_{\varphi} \dot{C} \equiv \mathscr{B} C+\mathscr{B} \dot{C}, \\
& Q_{d}=\mathscr{B} \bar{C}-p_{\varphi} \dot{\bar{C}} \equiv \mathscr{B} \bar{C}+\mathscr{B} \dot{\bar{C}},
\end{aligned}
$$

where the equivalent forms of the above charges are written with the help of the equation of motion: $p_{\varphi}=-\mathscr{B}$. The above charges are nilpotent of order two (i.e., $Q_{(a) b}^{2}=$ $\mathbb{Q}_{(a) d}^{2}=0$ ) and anticommuting in nature (i.e., $\mathbb{Q}_{b} Q_{a b}+\mathbb{Q}_{a b}$ $Q_{b}=0$ and $\left.Q_{d} Q_{a d}+Q_{a d} Q_{d}=0\right)$. The conservation law for these charges (i.e., $(d / d \tau) Q_{(a) b}=0$ and $\left.(d / d \tau) Q_{(a) d}=0\right)$ can be easily proven by using the following interesting EulerLagrange equations of motion [besides these EOMs, we use an equation $\ddot{\mathscr{B}}+\mathscr{B}=0$ derived from the EOMs (13) to prove the conservation law for the (anti-)BRST together with (anti-)co-BRST conserved charges (Equations (11) and (12))] (EOMs) derived from Lagrangian $L$ of our theory (Equation (7)), namely,

$$
\begin{gathered}
\mathscr{B}+p_{\varphi}=0, \\
\mathscr{B}=\dot{p}_{\varphi}, \\
\mathscr{B}=-(\dot{z}+\varphi), \\
\dot{p}_{r}-\frac{p_{\varphi}^{2}}{r^{3}}+V^{\prime}(r)=0, \\
\dot{r}=p_{r}, \\
\dot{\varphi}-z-\frac{p_{\varphi}}{r^{2}}=0, \\
\bar{C}+\ddot{\bar{C}}=0, \\
C+\ddot{C}=0 .
\end{gathered}
$$

The (anti-)co-BRST and (anti-)BRST conserved charges are the generators of the (anti-)co-BRST and (anti-)BRST symmetry transformations, respectively. As one can easily check that the following relationships are true:

$$
\begin{aligned}
s_{d} \xi & =-i\left[\xi, Q_{d}\right]_{ \pm}, \\
s_{a d} \xi & =-i\left[\xi, Q_{a d}\right]_{ \pm}, \\
s_{b} \xi & =-i\left[\xi, Q_{b}\right]_{ \pm}, \\
s_{a b} \xi & =-i\left[\xi, Q_{a b}\right]_{ \pm},
\end{aligned}
$$

where $\xi$ denotes any generic variable present in the Lagrangian $L$ of our theory. The subscript $( \pm)$ on the square brackets denotes the (anti)commutator which depends on the nature of generic variables $\xi$ being (fermionic) bosonic in nature.

\section{Nilpotent Quantum (Anti-)BRST Symmetry Transformations: (Anti-)chiral Supervariable Approach}

In this section, we determine the nilpotent (anti-)BRST symmetry transformations (cf. Equation (8)) by using (anti-)chiral supervariable approach (ACSA) to BRST formalism where we shall use the (anti-)chiral super expansions of supervariables. Towards this goal, first of all, we generalize the ordinary variables of the Lagrangian $(7)$ onto $(1,1)$ -dimensional antichiral super-submanifold (of the most common (1,2)-dimensional supermanifold) as follows:

$$
\begin{aligned}
z(\tau) \longrightarrow \mathscr{Z}(\tau, \bar{\vartheta}) & =z(\tau)+\bar{\vartheta} f_{1}(\tau), \\
\varphi(\tau) \longrightarrow \Theta(\tau, \bar{\vartheta}) & =\varphi(\tau)+\bar{\vartheta} f_{2}(\tau), \\
C(\tau) \longrightarrow F(\tau, \bar{\vartheta}) & =C(\tau)+i \bar{\vartheta} b_{1}(\tau), \\
\bar{C}(\tau) \longrightarrow F(\tau, \bar{\vartheta}) & =\bar{C}(\tau)+i \bar{\vartheta} b_{2}(\tau), \\
r(\tau) \longrightarrow & R(\tau, \bar{\vartheta})=r(\tau)+\bar{\vartheta} f_{3}(\tau), \\
p_{r}(\tau) \longrightarrow P_{r}(\tau, \bar{\vartheta}) & =p_{r}(\tau)+\bar{\vartheta} f_{4}(\tau), \\
p_{\varphi}(\tau) \longrightarrow P_{\varphi}(\tau, \bar{\vartheta}) & =p_{\varphi}(\tau)+\bar{\vartheta} f_{5}(\tau), \\
\mathscr{B}(\tau) \longrightarrow \tilde{\mathscr{B}}(\tau, \bar{\vartheta}) & =\mathscr{B}(\tau)+\bar{\vartheta} f_{6}(\tau),
\end{aligned}
$$

where $b_{1}, b_{2}$ are the bosonic derived variables and $f_{1}, f_{2}$ , $f_{3}, f_{4}, f_{5}, f_{6}$ are the fermionic derived variables due to fermionic nature of $\bar{\vartheta}$. We determine the precise value of these derived variables in terms of the auxiliary and basic variables present in the BRST invariant Lagrangian (7) by using the BRST invariant quantities/restrictions.

According to the basic principles of ACSA, the BRST invariant quantities must remain independent of the Grassmannian variable $(\bar{\vartheta})$ when they are generalized onto the ( 1,1)-dimensional antichiral super-submanifold. The BRST invariant quantities are the specific combinations of the variables present in Lagrangian (7). These are given as follows:

$$
\begin{aligned}
s_{b}\left(r, p_{r}, p_{\varphi}, \mathscr{B}, C\right) & =0, \\
s_{b}(z \dot{C}) & =0, \\
s_{b}(\varphi C) & =0, \\
s_{b}(\mathscr{B} z+i \dot{\bar{C}} \dot{C}) & =0, \\
s_{b}(\dot{\varphi}-z) & =0, \\
s_{b}(\mathscr{B} \varphi+i \bar{C} C) & =0 .
\end{aligned}
$$

We generalize the above BRST invariant restrictions onto the $(1,1)$-dimensional antichiral super-submanifolds (of the suitably chosen most common $(1,2)$-dimensional supermanifold) 


$$
\begin{aligned}
& R(\tau, \bar{\vartheta})=r(\tau), P_{r}(\tau, \bar{\vartheta})=p_{r}(\tau), P_{\varphi}(\tau, \bar{\vartheta})=p_{\varphi}(\tau), \\
& \tilde{\mathscr{B}}(\tau, \bar{\vartheta})=\mathscr{B}(\tau), F(\tau, \bar{\vartheta})=C(\tau), \\
& \mathscr{Z}(\tau, \bar{\vartheta}) \dot{F}(\tau, \bar{\vartheta})=z(\tau) \dot{C}(\tau), \Theta(\tau, \bar{\vartheta}) F(\tau, \bar{\vartheta})=\varphi(\tau) C(\tau),
\end{aligned}
$$

$$
\dot{\mathscr{\mathscr { B }}}(\tau, \bar{\vartheta}) \mathscr{Z}(\tau, \bar{\vartheta})+i \dot{\bar{F}}(\tau, \bar{\vartheta}) \dot{C}(\tau, \bar{\vartheta})=\dot{\mathscr{B}}(\tau) z(\tau)+i \dot{\bar{C}}(\tau) \dot{C}(\tau)
$$

$$
\dot{\Theta}(\tau, \bar{\vartheta})-\mathscr{Z}(\tau, \bar{\vartheta})=\dot{\varphi}(\tau)-z(\tau),
$$

$\tilde{\mathscr{B}}(\tau, \bar{\vartheta}) \Theta(\tau, \bar{\vartheta})+i \bar{F}(\tau, \bar{\vartheta}) F(\tau, \bar{\vartheta})=\mathscr{B}(\tau) \varphi(\tau)+i \bar{C}(\tau) C(\tau)$

The above restrictions lead to the derivation of the derived variables in terms of the basic and auxiliary variables. To determine the value of these variables, we perform the step-by-step explicit calculations. For this purpose, first of all, we use the generalization of the trivial BRST invariant restrictions given in the first line of Equation (24) as

$$
\begin{gathered}
P_{\varphi}(\tau, \bar{\vartheta})=p_{\varphi}(\tau) \Rightarrow f_{5}=0, \\
\tilde{\mathscr{B}}(\tau, \bar{\vartheta})=\mathscr{B}(\tau) \Rightarrow f_{6}=0, \\
R(\tau, \bar{\vartheta})=r(\tau) \Rightarrow f_{3}=0, \\
F(\tau, \bar{\vartheta})=C(\tau) \Rightarrow b_{1}=0, \\
P_{r}(\tau, \bar{\vartheta})=p_{r}(\tau) \Rightarrow f_{4}=0 .
\end{gathered}
$$

After substituting the above value of derived variables from (29) to (15), we get the following expressions for the antichiral supervariables, namely,

$$
\begin{gathered}
C(\tau) \longrightarrow F^{(b)}(\tau, \bar{\vartheta})=C(\tau)+\bar{\vartheta}(0) \equiv C(\tau)+\bar{\vartheta}\left[s_{b} C(\tau)\right], \\
r(\tau) \longrightarrow R^{(b)}(\tau, \bar{\vartheta})=r(\tau)+\bar{\vartheta}(0) \equiv r(\tau)+\bar{\vartheta}\left[s_{b} r(\tau)\right], \\
p_{r}(\tau) \longrightarrow P_{r}^{(b)}(\tau, \bar{\vartheta})=p_{r}(\tau)+\bar{\vartheta}(0) \equiv p_{r}(\tau)+\bar{\vartheta}\left[s_{b} p_{r}(\tau)\right], \\
p_{\varphi}(\tau) \longrightarrow P_{\varphi}^{(b)}(\tau, \bar{\vartheta})=p_{\varphi}(\tau)+\bar{\vartheta}(0) \equiv p_{\varphi}(\tau)+\bar{\vartheta}\left[s_{b} p_{\varphi}(\tau)\right], \\
\mathscr{B}(\tau) \longrightarrow \tilde{\mathscr{B}}^{(b)}(\tau, \bar{\vartheta})=\mathscr{B}(\tau)+\bar{\vartheta}(0) \equiv \mathscr{B}(\tau)+\bar{\vartheta}\left[s_{b} \mathscr{B}(\tau)\right],
\end{gathered}
$$

where the superscript $(b)$ on the antichiral supervariables denotes that these supervariables have been obtained after the use of BRST invariant quantities. It is clear that the coefficients of Grassmannian variable $\bar{\vartheta}$ are simply the quantum BRST symmetries (8). Now, in the case of nontrivial BRST invariant restrictions: $s_{b}(z \dot{C})=0$ and $s_{b}(\varphi C)=0$, the following generalizations onto $(1,1)$-dimensional super-submanifold, namely,

$$
\mathscr{Z}(\tau, \bar{\vartheta}) \dot{F}^{(b)}(\tau, \bar{\vartheta})=z(\tau) \dot{C}(\tau), \Theta(\tau, \bar{\vartheta}) F^{(b)}(\tau, \bar{\vartheta})=\varphi(\tau) C(\tau),
$$

lead to the following interesting results:

$$
\begin{aligned}
f_{1}(\tau) \dot{C}(\tau) & =0 \Rightarrow f_{1}(\tau) \propto \dot{C}(\tau), \Rightarrow f_{1}(\tau)=\kappa_{1} \dot{C}(\tau), \\
f_{2}(\tau) C(\tau)=0 & \Rightarrow f_{2}(\tau) \propto C(\tau), \Rightarrow f_{2}(\tau)=\kappa_{2} C(\tau),
\end{aligned}
$$

where $\kappa_{1}$ and $\kappa_{2}$ are the proportionality constants. To determine the value of these constants, we use the generalization of BRST invariant restriction $s_{b}(\dot{\varphi}-z)=0$ as

$$
\dot{\Theta}(\tau, \bar{\vartheta})-\mathscr{Z}(\tau, \bar{\vartheta})=\dot{\varphi}(\tau)-z(\tau) \Rightarrow \kappa_{1}=\kappa_{2} .
$$

Finally, to determine the value of constants, we generalize the BRST invariant restrictions $s_{b}(\dot{\mathscr{B}} z+i \dot{\bar{C}} \dot{C})=0$ and $s_{b}(\dot{\mathscr{B}} \varphi$ $+i \bar{C} C)=0$ onto $(1,1)$-dimensional super-submanifold as

$$
\begin{aligned}
& \dot{\mathscr{\mathscr { B }}}^{(b)}(\tau, \bar{\vartheta}) \mathscr{Z}(\tau, \bar{\vartheta})+i \dot{\bar{F}}(\tau, \bar{\vartheta}) \dot{F}^{(b)}(\tau, \bar{\vartheta}) \\
& \quad=\dot{\mathscr{B}}(\tau) z(\tau)+i \dot{\bar{C}}(\tau) \dot{C}(\tau) \Rightarrow b_{2}(\tau)=\kappa_{1} \dot{\mathscr{B}}(\tau), \\
& \quad \tilde{\mathscr{B}}^{(b)}(\tau, \bar{\vartheta}) \Theta(\tau, \bar{\vartheta})+i \bar{F}(\tau, \overline{9}) F^{(b)}(\tau, \bar{\vartheta}) \\
& =\mathscr{B}(\tau) \varphi(\tau)+i \bar{C}(\tau) C(\tau) \Rightarrow b_{2}(\tau)=\kappa_{2} \mathscr{B}(\tau) .
\end{aligned}
$$

Using the results obtained in Equations (33) and (34), it is clear that $\kappa_{1}=\kappa_{2}=1$. Therefore, we obtain the value of derived variables as $f_{1}(\tau)=\dot{C}(\tau), f_{2}(\tau)=C(\tau), b_{2}(\tau)=\mathscr{B}(\tau)$; thus, we get the following expansions for antichiral supervariables:

$$
z(\tau) \longrightarrow \mathscr{Z}^{(b)}(\tau, \bar{\vartheta})=z(\tau)+\bar{\vartheta}[\dot{C}(\tau)] \equiv z(\tau)+\bar{\vartheta}\left[s_{b} z(\tau)\right],
$$

$$
\varphi(\tau) \longrightarrow \Theta^{(b)}(\tau, \bar{\vartheta})=\varphi(\tau)+\bar{\vartheta}[C(\tau)] \equiv \varphi(\tau)+\bar{\vartheta}\left[s_{b} \varphi(\tau)\right]
$$

$$
\bar{C}(\tau) \longrightarrow \bar{F}^{(b)}(\tau, \bar{\vartheta})=\bar{C}(\tau)+\bar{\vartheta}[i \mathscr{B}(\tau)] \equiv \bar{C}(\tau)+\bar{\vartheta}\left[s_{b} \bar{C}(\tau)\right]
$$

Thus, in view of Equation (36), we have a connection between the BRST symmetry transformation $\left(s_{b}\right)$ and partial derivative $\left(\partial_{\bar{\vartheta}}\right)$ on the antichiral super-submanifold defined by the mapping: $s_{b} \leftrightarrow \partial_{\overline{9}}$ (see, e.g., [11-13] for details). To be more clear, the BRST transformation of any generic variable $\psi(\tau)$ is equal to the translation of the corresponding antichiral supervariable $\Psi^{(b)}(\tau, \bar{\vartheta})$ along the $\overline{9}$-direction. Mathematically, it can be represented as $s_{b} \psi(\tau)=(\partial / \partial \bar{\vartheta}) \Psi^{(b)}(\tau, \bar{\vartheta})=\partial_{\bar{\vartheta}}$ $\Psi^{(b)}(\tau, \bar{\vartheta})$. In other words, one can say that the coefficient of $\bar{\vartheta}$ in the expansion of an antichiral supervariable is simply the quantum BRST symmetry transformation of the corresponding variable.

We are now in the stage to derive the quantum antiBRST symmetry transformations using chiral supervariable approach. In this context, we use the chiral super expansions of the chiral supervariables where we generalize $(0+1)$ -dimensional variables onto the $(1,1)$-dimensional supersubmanifold of the suitably chosen most common $(1,2)$ 
-dimensional supermanifold. The chiral super expansions of the ordinary variables are as follows:

$$
\begin{aligned}
z(\tau) \longrightarrow \mathscr{Z}(\tau, \vartheta) & =z(\tau)+\vartheta \bar{f}_{1}(\tau), \\
\varphi(\tau) \longrightarrow \Theta(\tau, \vartheta) & =\varphi(\tau)+\vartheta \bar{f}_{2}(\tau) \\
C(\tau) \longrightarrow F(\tau, \vartheta) & =C(\tau)+i \vartheta \bar{b}_{1}(\tau), \\
\bar{C}(\tau) \longrightarrow \bar{F}(\tau, \vartheta) & =\bar{C}(\tau)+i \vartheta \bar{b}_{2}(\tau), \\
r(\tau) \longrightarrow R(\tau, \vartheta) & =r(\tau)+\vartheta \bar{f}_{3}(\tau), \\
p_{r}(\tau) \longrightarrow P_{r}(\tau, \vartheta) & =p_{r}(\tau)+\vartheta \bar{f}_{4}(\tau), \\
p_{\varphi}(\tau) \longrightarrow P_{\varphi}(\tau, \vartheta) & =p_{\varphi}(\tau)+\vartheta \bar{f}_{5}(\tau), \\
\mathscr{B}(\tau) \longrightarrow \tilde{\mathscr{B}}(\tau, \vartheta) & =\mathscr{B}(\tau)+\vartheta \bar{f}_{6}(\tau),
\end{aligned}
$$

where derived variables $\bar{b}_{1}, \bar{b}_{2}$ are the bosonic and derived variables $\bar{f}_{1}, \bar{f}_{2}, \bar{f}_{3}, \bar{f}_{4}, \bar{f}_{5}, \bar{f}_{6}$ are fermionic in nature. The antiBRST invariant restrictions also must remain independent of the Grassmannian variable ( $\vartheta$ ) when they are generalized onto the $(1,1)$-dimensional chiral super-submanifold. The antiBRST invariant restrictions are given as

$$
\begin{gathered}
s_{a b}\left(r, p_{r}, p_{\varphi}, \mathscr{B}, \bar{C}\right)=0, \\
s_{a b}(z \dot{\bar{C}})=0, \\
s_{a b}(\varphi \bar{C})=0, \\
s_{a b}(\dot{\mathscr{B}} z+i \dot{\bar{C}} \dot{C})=0, \\
s_{a b}(\dot{\varphi}-z)=0, \\
s_{a b}(\mathscr{B} \varphi+i \bar{C} C)=0 .
\end{gathered}
$$

As the physical quantities remain independent of the Grassmannian variable $\vartheta$ which imply that the anti-BRST invariant restrictions can be generalized onto the $(1,1)$ -dimensional super-submanifold of the most common $(1,2)$ -dimensional supermanifold as follows:

$$
\begin{gathered}
R(\tau, \vartheta)=r(\tau), P_{r}(\tau, \vartheta)=p_{r}(\tau), P_{\varphi}(\tau, \vartheta)=p_{\varphi}(\tau), \\
\tilde{\mathscr{B}}(\tau, \vartheta)=\mathscr{B}(\tau), \bar{F}(\tau, \vartheta)=\bar{C}(\tau), \\
\mathscr{Z}(\tau, \vartheta) \dot{\bar{F}}(\tau, \vartheta)=z(\tau) \dot{\bar{C}}(\tau), \Theta(\tau, \vartheta) \bar{F}(\tau, \vartheta)=\varphi(\tau) \bar{C}(\tau), \\
\dot{\tilde{B}}(\tau, \vartheta) Z(\tau, \vartheta)+i \dot{\bar{F}}(\tau, \vartheta) \dot{F}(\tau, \vartheta)=\dot{\mathscr{B}}(\tau) z(\tau)+i \dot{\bar{C}}(\tau) \dot{C}(\tau), \\
\dot{\Theta}(\tau, \vartheta)-\mathscr{Z}(\tau, \vartheta)=\dot{\varphi}(\tau)-z(\tau), \\
\tilde{\mathscr{B}}(\tau, \vartheta) \Theta(\tau, \vartheta)+i \bar{F}(\tau, \vartheta) F(\tau, \vartheta)=\mathscr{B}(\tau) \varphi(\tau)+i \bar{C}(\tau) C(\tau)
\end{gathered}
$$

The above generalizations of the anti-BRST invariant restrictions (Equation (47)) lead to the derivation of the chiral derived variables in terms of the auxiliary and basic variables present in the Lagrangian $L$, namely,

$$
\begin{aligned}
& \bar{b}_{2}=0, \\
& \bar{f}_{3}=0, \\
& \bar{f}_{4}=0, \\
& \bar{f}_{5}=0, \\
& \bar{f}_{6}=0, \\
& \bar{f}_{1}=\dot{\bar{C}}, \\
& \bar{f}_{2}=\bar{C}, \\
& \bar{b}_{1}=-\mathscr{B} .
\end{aligned}
$$

The above values of chiral derived variables are obtained in a similar fashion as the antichiral derived variables are obtained. After the substitution of the above derived variables into the chiral super expansions (39), we get the following expressions for the chiral supervariables onto $(1,1)$-dimensional super-submanifold as

$$
\begin{gathered}
z(\tau) \longrightarrow \mathscr{Z}^{(a b)}(\tau, \vartheta)=z(\tau)+\vartheta(\dot{\bar{C}}) \equiv z(\tau)+\vartheta\left[s_{a b} z(\tau)\right] \\
\varphi(\tau) \longrightarrow \Theta^{(a b)}(\tau, \vartheta)=\varphi(\tau)+\vartheta(\bar{C}) \equiv \varphi(\tau)+\vartheta\left[s_{a b} \varphi(\tau)\right] \\
C(\tau) \longrightarrow F^{(a b)}(\tau, \vartheta)=C(\tau)+\vartheta(-i \mathscr{B}) \equiv C(\tau)+\vartheta\left[s_{a b} C(\tau)\right] \\
\bar{C}(\tau) \longrightarrow \bar{F}^{(a b)}(\tau, \vartheta)=\bar{C}(\tau)+\vartheta(0) \equiv \bar{C}(\tau)+\vartheta\left[s_{a b} \bar{C}(\tau)\right], \\
r(\tau) \longrightarrow R^{(a b)}(\tau, \vartheta)=r(\tau)+\vartheta(0) \equiv r(\tau)+\vartheta\left[s_{a b} r(\tau)\right], \\
p_{r}(\tau) \longrightarrow P_{r}^{(a b)}(\tau, \vartheta)=p_{r}(\tau)+\vartheta(0) \equiv p_{r}(\tau)+\vartheta\left[s_{a b} p_{r}(\tau)\right] \\
p_{\varphi}(\tau) \longrightarrow P_{\varphi}^{(a b)}(\tau, \vartheta)=p_{\varphi}(\tau)+\vartheta(0) \equiv p_{\varphi}(\tau)+\vartheta\left[s_{a b} p_{\varphi}(\tau)\right], \\
\mathscr{B}(\tau) \longrightarrow \tilde{\mathscr{B}}^{(a b)}(\tau, \vartheta)=\mathscr{B}(\tau)+\vartheta(0) \equiv \mathscr{B}(\tau)+\vartheta\left[s_{a b} \mathscr{B}(\tau)\right]
\end{gathered}
$$

Here, the coefficients of $\vartheta$ are simply the anti-BRST symmetry transformations (see, e.g., [11-13] for detail). In fact, the anti-BRST transformation of any generic variable $\psi(\tau)$ is simply the translation of the corresponding chiral supervariable $\Psi^{(a b)}(\tau, \vartheta)$ along the $\vartheta$-direction. Mathematically, this statement can be corroborated as $s_{a b} \psi(\tau)=(\partial / \partial \vartheta) \Psi^{(a b)}(\tau, \vartheta)$ $=\partial_{\vartheta} \Psi^{(a b)}(\tau, \vartheta)$. Thus, it is clear that there is a mapping between the quantum anti-BRST symmetry transformation ( $\left.s_{a b}\right)$ and the Grassmannian partial derivative $\left(\partial_{\vartheta}\right)$ defined on the chiral super-submanifold with the mapping: $s_{a b} \leftrightarrow \partial_{\vartheta}$. 


\section{Nilpotent (Anti-)co-BRST Symmetry Transformations: (Anti-)chiral Supervariable Approach}

In this section, we derive the nilpotent (anti-)co-BRST symmetry transformations using the (anti-)chiral supervariable approach (ACSA) where we use the expansions of the (anti-)chiral supervariables and the (anti-)co-BRST invariant restrictions. Toward this goal in our mind, first of all, we determine the co-BRST symmetries by exploiting the chiral super expansions given in Equation (39) and the coBRST invariant restrictions. The co-BRST invariant restrictions are given as

$$
\begin{aligned}
s_{d}\left(r, p_{r}, p_{\varphi}, \mathscr{B}, \bar{C}\right) & =0, \\
s_{d}(z \bar{C}) & =0, \\
s_{d}(\varphi \dot{\bar{C}}) & =0, \\
s_{d}\left(\varphi \dot{p}_{\varphi}+i \dot{\bar{C}} \dot{C}\right) & =0, \\
s_{d}\left(z p_{\varphi}-i \bar{C} C\right) & =0, \\
s_{d}(\varphi+\dot{z}) & =0 .
\end{aligned}
$$

According to the basic rules of ACSA, the above coBRST invariant restrictions can be generalized onto the (1, 1)-dimensional super-submanifold (of the suitably chosen most common $(1,2)$-dimensional supermanifold) as

$$
\begin{aligned}
& R(\tau, \vartheta)=r(\tau), P_{r}(\tau, \vartheta)=p_{r}(\tau), P_{\varphi}(\tau, \vartheta)=p_{\varphi}(\tau), \\
& \tilde{\mathscr{B}}(\tau, \vartheta)=\mathscr{B}(\tau), \bar{F}(\tau, \vartheta)=\bar{C}(\tau),
\end{aligned}
$$

$$
\dddot{Z}(\tau, \vartheta) \bar{F}(\tau, \vartheta)=z(\tau) \bar{C}(\tau), \Theta(\tau, \vartheta) \dot{\bar{F}}(\tau, \vartheta)=\varphi(\tau) \dot{\bar{C}}(\tau),
$$

$\Theta(\tau, \vartheta) \dot{P}_{\varphi}(\tau, \vartheta)+i \dot{\bar{F}}(\tau, \vartheta) \dot{F}(\tau, \vartheta)=\varphi(\tau) \dot{p}_{\varphi}(\tau)+i \dot{\bar{C}}(\tau) \dot{C}(\tau)$

$\mathscr{Z}(\tau, \vartheta) P_{\varphi}(\tau, \vartheta)-i \bar{F}(\tau, \vartheta) F(\tau, \vartheta)=z(\tau) p_{\varphi}(\tau)-i \bar{C}(\tau) C(\tau)$,

$$
\Theta(\tau, \vartheta)+\dot{Z}(\tau, \vartheta)=\varphi(\tau)+\dot{z}(\tau) .
$$

At this stage, we determine the value of derived variables of Equation (39) using the above generalizations of the co-BRST invariant restrictions. To derive the value of the derived variables, first of all, we use the first line entry of Equation (52) where the trivial co-BRST invariant quantities are generalized which implies the following relationships:

$$
\begin{gathered}
P_{\varphi}(\tau, \vartheta)=p_{\varphi}(\tau) \Rightarrow \bar{f}_{5}=0, \\
\tilde{\mathscr{B}}(\tau, \vartheta)=\mathscr{B}(\tau) \Rightarrow \bar{f}_{6}=0, \\
R(\tau, \vartheta)=r(\tau) \Rightarrow \bar{f}_{3}=0, \\
\bar{F}(\tau, \vartheta)=\bar{C}(\tau) \Rightarrow \bar{b}_{2}=0, \\
P_{r}(\tau, \vartheta)=p_{r}(\tau) \Rightarrow \bar{f}_{4}=0 .
\end{gathered}
$$

After substituting the above value of derived variables into the expressions of the chiral super expansions (Equation (39)), we obtain the following chiral super expansions:

$$
\begin{array}{r}
\bar{C}(\tau) \longrightarrow \bar{F}^{(d)}(\tau, \vartheta)=\bar{C}(\tau)+\vartheta(0) \equiv \bar{C}(\tau)+\vartheta\left[s_{d} \bar{C}(\tau)\right], \\
r(\tau) \longrightarrow R^{(d)}(\tau, \vartheta)=r(\tau)+\vartheta(0) \equiv r(\tau)+\vartheta\left[s_{d} r(\tau)\right], \\
p_{r}(\tau) \longrightarrow P_{r}^{(d)}(\tau, \vartheta)=p_{r}(\tau)+\vartheta(0) \equiv p_{r}(\tau)+\vartheta\left[s_{d} p_{r}(\tau)\right], \\
p_{\varphi}(\tau) \longrightarrow P_{\varphi}^{(d)}(\tau, \vartheta)=p_{\varphi}(\tau)+\vartheta(0) \equiv p_{\varphi}(\tau)+\vartheta\left[s_{d} p_{\varphi}(\tau)\right], \\
\mathscr{B}(\tau) \longrightarrow \tilde{\mathscr{B}}^{(d)}(\tau, \vartheta)=\mathscr{B}(\tau)+\vartheta(0) \equiv \mathscr{B}(\tau)+\vartheta\left[s_{d} \mathscr{B}(\tau)\right],
\end{array}
$$

where superscript $(d)$ on the chiral supervariables denotes the supervariables obtained after the application of the co-BRST (i.e., dual-BRST) invariant restrictions. For the nontrivial case, first of all, we generalize the coBRST invariant restriction $s_{d}(z \bar{C})=0$ and $s_{d}(\varphi \dot{\bar{C}})=0$ onto $(1,1)$-dimensional super-submanifold as

$\mathscr{Z}(\tau, \vartheta) \bar{F}^{(d)}(\tau, \vartheta)=z(\tau) \bar{C}(\tau), \Theta(\tau, \vartheta) \dot{\bar{F}}^{(d)}(\tau, \vartheta)=\varphi(\tau) \dot{\bar{C}}(\tau)$,

which lead to the following relationships for the derived variables:

$$
\begin{gathered}
\bar{f}_{1}(\tau) \bar{C}(\tau)=0 \Rightarrow \bar{f}_{1}(\tau) \propto \bar{C}(\tau), \Rightarrow \bar{f}_{1}(\tau)=-\bar{\kappa}_{1} \bar{C}(\tau), \\
\bar{f}_{2}(\tau) \dot{\bar{C}}(\tau)=0 \Rightarrow \bar{f}_{2}(\tau) \propto \dot{\bar{C}}(\tau), \Rightarrow \overline{\mathrm{f}}_{2}(\tau)=\bar{\kappa}_{2} \dot{\bar{C}}(\tau),
\end{gathered}
$$

where $\bar{\kappa}_{1}$ and $\bar{\kappa}_{2}$ are the proportionality constants. To determine the value of these constants, we further use the generalizations of the co-BRST invariant restrictions $s_{d}(\varphi+\dot{z})=0, s_{d}\left(\varphi \dot{p}_{\varphi}+i \dot{\bar{C}} \dot{C}\right)=0$ and $s_{d}\left(z p_{\varphi}-i \bar{C} C\right)=0$ as

$$
\begin{gathered}
\Theta(\tau, \vartheta)+\dot{\mathscr{Z}}(\tau, \vartheta)=\varphi(\tau)+\dot{z}(\tau) \Rightarrow \bar{\kappa}_{1}=\bar{\kappa}_{2}, \\
\Theta(\tau, \vartheta) \dot{P}_{\varphi}^{(d)}(\tau, \vartheta)+i \dot{\bar{F}}^{(d)}(\tau, \vartheta) \dot{F}(\tau, \vartheta)=\varphi(\tau) \dot{p}_{\varphi}(\tau) \\
+i \dot{\bar{C}}(\tau) \dot{C}(\tau) \Rightarrow \dot{\bar{b}}_{1}(\tau)=-\bar{\kappa}_{2} \dot{p}_{\varphi}(\tau), \\
\mathscr{Z}(\tau, \vartheta) P_{\varphi}^{(d)}(\tau, \vartheta)-i \bar{F}^{(d)}(\tau, \vartheta) F(\tau, \vartheta)=z(\tau) p_{\varphi}(\tau) \\
-i \bar{C}(\tau) C(\tau) \Rightarrow \bar{b}_{1}(\tau)=-\bar{\kappa}_{1} p_{\varphi}(\tau) .
\end{gathered}
$$

The results of the above three relations in Equation (65) imply that proportionality constant is equal (i.e., $\bar{\kappa}_{1}$ 
$=\bar{\kappa}_{2}$ ) and their values are equal to minus one (i.e., $\bar{\kappa}_{1}=$ $\left.\bar{\kappa}_{2}=-1\right)$. Therefore, we get the value of the derived variables as $\bar{f}_{1}=\bar{C}, \bar{f}_{2}=-\dot{\bar{C}}, \bar{b}_{1}=p_{\varphi}$. As a result, we have the following chiral super expansions of the ordinary variables:

$$
\begin{gathered}
z(\tau) \longrightarrow \mathscr{Z}^{(d)}(\tau, \vartheta)=z(\tau)+\vartheta[\bar{C}(\tau)] \equiv z(\tau)+\vartheta\left[s_{d} z(\tau)\right] \\
\varphi(\tau) \longrightarrow \Theta^{(d)}(\tau, \vartheta)=\varphi(\tau)+\vartheta[-\dot{\bar{C}}(\tau)] \equiv \varphi(\tau)+\vartheta\left[s_{d} \varphi(\tau)\right] \\
C(\tau) \longrightarrow F^{(d)}(\tau, \vartheta)=C(\tau)+\vartheta\left[i p_{\varphi}(\tau)\right] \equiv C(\tau)+\vartheta\left[s_{d} C(\tau)\right]
\end{gathered}
$$

where superscript $(d)$ on the supervariables denotes the same meaning as in Equation (58). From the above equations, it is clear that the translation of any generic chiral supervariable $\Psi^{(d)}(\tau, \vartheta)$ along the $\vartheta$-direction generates co-BRST symmetry transformation $\left(s_{d}\right)$ of the corresponding variable $\psi(\tau)$. Mathematically, we can express these statements as $s_{d} \psi(\tau)=\partial_{\vartheta} \Psi^{(d)}(\tau, \vartheta)$. In other words, the coefficients of $\vartheta$ are simply the co-BRST symmetry transformations. Thus, the co-BRST symmetry transformation $\left(s_{d}\right)$ is connected with the Grassmannian derivative $\partial_{\vartheta}$ (i.e., $\left.s_{d} \leftrightarrow \partial_{\vartheta}\right)[11-13]$.

Now, for the derivation of anti-co-BRST symmetry transformations, we use the antichiral super expansions of the supervariables (Equation (15)) and anti-co-BRST invariant restrictions. The anti-co-BRST invariant restrictions are listed as

$$
\begin{aligned}
s_{a d}\left(r, p_{r}, p_{\varphi}, \mathscr{B}, C\right) & =0, \\
s_{a d}(z C) & =0, \\
s_{a d}(\varphi \dot{C}) & =0, \\
s_{a d}\left(z p_{\varphi}-i \bar{C} C\right) & =0, \\
s_{a d}\left(\varphi \dot{p}_{\varphi}+i \dot{\bar{C}} \dot{C}\right) & =0, \\
s_{a d}(\dot{z}+\varphi) & =0 .
\end{aligned}
$$

The generalization of these anti-co-BRST invariant restrictions onto the $(1,1)$-dimensional super-submanifold (of the most common $(1,2)$-dimensional supermanifold) is as follows:

$$
\begin{gathered}
R(\tau, \bar{\vartheta})=r(\tau), P_{r}(\tau, \bar{\vartheta})=p_{r}(\tau), P_{\varphi}(\tau, \bar{\vartheta})=p_{\varphi}(\tau), \\
\tilde{\mathscr{B}}(\tau, \bar{\vartheta})=\mathscr{B}(\tau), F(\tau, \bar{\vartheta})=C(\tau), \\
\mathscr{Z}(\tau, \bar{\vartheta}) F(\tau, \bar{\vartheta})=z(\tau) C(\tau), \Theta(\tau, \bar{\vartheta}) \dot{F}(\tau, \bar{\vartheta})=\varphi(\tau) \dot{C}(\tau), \\
\mathscr{Z}(\tau, \bar{\vartheta}) P_{\varphi}(\tau, \bar{\vartheta})-i \bar{F}(\tau, \bar{\vartheta}) F(\tau, \bar{\vartheta})=z(\tau) p_{\varphi}(\tau)-i \bar{C}(\tau) C(\tau), \\
\Theta(\tau, \bar{\vartheta}) \dot{P}_{\varphi}(\tau, \bar{\vartheta})+i \dot{\bar{F}}(\tau, \bar{\vartheta}) \dot{F}(\tau, \bar{\vartheta})=\varphi(\tau) p_{\varphi}(\tau)+i \dot{\bar{C}}(\tau) \dot{C}(\tau), \\
\dot{\mathscr{Z}}(\tau, \bar{\vartheta})+\Theta(\tau, \bar{\vartheta})=\dot{z}(\tau)+\varphi(\tau) .
\end{gathered}
$$

The above generalizations of the anti-co-BRST invariant restrictions, finally, lead to the derivation of the derived variables as follows:

$$
\begin{gathered}
b_{1}=0, \\
b_{2}=-p_{\varphi}, \\
f_{1}=C, \\
f_{2}=-\dot{C}, \\
f_{3}=0, \\
f_{4}=0, \\
f_{5}=0, \\
f_{6}=0 .
\end{gathered}
$$

Thus, we have determined all the derived variables using the same technique as we have used in the derivation of the coBRST symmetry transformations. Finally, after substituting the value of derived variables into Equation (15), we obtain the following expressions for the antichiral expansions of the supervariables, namely,

$$
\begin{gathered}
z(\tau) \longrightarrow \mathscr{Z}^{(a d)}(\tau, \bar{\vartheta})=z(\tau)+\bar{\vartheta}(C) \equiv z(\tau)+\bar{\vartheta}\left[s_{a d} z(\tau)\right] \\
\varphi(\tau) \longrightarrow \Theta^{(a d)}(\tau, \bar{\vartheta})=\varphi(\tau)+\bar{\vartheta}(-\dot{C}) \equiv \varphi(\tau)+\bar{\vartheta}\left[s_{a d} \varphi(\tau)\right] \\
C(\tau) \longrightarrow F^{(a d)}(\tau, \bar{\vartheta})=C(\tau)+\bar{\vartheta}(0) \equiv C(\tau)+\bar{\vartheta}\left[s_{a d} C(\tau)\right] \\
\bar{C}(\tau) \longrightarrow \bar{F}^{(a d)}(\tau, \bar{\vartheta})=\bar{C}(\tau)+\bar{\vartheta}\left(-i p_{\varphi}\right) \equiv \bar{C}(\tau)+\bar{\vartheta}\left[s_{a d} \bar{C}(\tau)\right] \\
r(\tau) \longrightarrow R^{(a d)}(\tau, \bar{\vartheta})=r(\tau)+\bar{\vartheta}(0) \equiv r(\tau)+\bar{\vartheta}\left[s_{a d} r(\tau)\right] \\
p_{r}(\tau) \longrightarrow P_{r}^{(a d)}(\tau, \bar{\vartheta})=p_{r}(\tau)+\bar{\vartheta}(0) \equiv p_{r}(\tau)+\bar{\vartheta}\left[s_{a d} p_{r}(\tau)\right] \\
p_{\varphi}(\tau) \longrightarrow P_{\varphi}^{(a d)}(\tau, \bar{\vartheta})=p_{\varphi}(\tau)+\bar{\vartheta}(0) \equiv p_{\varphi}(\tau)+\bar{\vartheta}\left[s_{a d} p_{\varphi}(\tau)\right] \\
\mathscr{B}(\tau) \longrightarrow \tilde{\mathscr{B}}^{(a d)}(\tau, \bar{\vartheta})=\mathscr{B}^{(\tau)}(\tau)+\bar{\vartheta}(0) \equiv \mathscr{B}(\tau)+\bar{\vartheta}\left[s_{a d} \mathscr{B}(\tau)\right]
\end{gathered}
$$

where superscript $(a d)$ on the antichiral supervariables denotes the fact that antichiral supervariables are obtained after the application of quantum anti-co-BRST invariant restrictions (Equation (69)). Here, it is clear that the coefficient of Grassmannian variable $\bar{\vartheta}$ is simply the quantum anti-co-BRST symmetry transformation $\left(s_{a d}\right)$. To be more clear, the anti-coBRST symmetry transformation $\left(s_{a d}\right)$ of any generic variable $\psi(\tau)$ is equal to the translation of the corresponding antichiral supervariable $\Psi^{(a d)}(\tau, \bar{\vartheta})$ along the $\bar{\vartheta}$-direction, i.e., $s_{a d} \psi(\tau)=$ $\partial_{\bar{\vartheta}} \Psi^{(a d)}(\tau, \bar{\vartheta})$. This implies that the anti-co-BRST symmetry $\left(s_{a d}\right)$ is connected with the Grassmannian translation generator $\left(\partial_{\bar{\vartheta}}\right)$ as $s_{a d} \leftrightarrow \partial_{\bar{\vartheta}}[11-13]$. 


\section{Nilpotency and Absolute Anticommutativity of the Noether Conserved Charges: ACSA}

In this section, we deduce the nilpotency and absolute anticommutativity properties of the conserved (anti-)BRST along with (anti-)co-BRST charges in the language of ACSA. For this, first of all, we show the nilpotency of the (anti-)BRST together with (anti-)co-BRST conserved charges. It is straightforward to express the expressions of the (anti)BRST together with (anti-)co-BRST charges in terms of the (anti-)chiral supervariables and partial derivatives $\left(\partial_{\bar{\vartheta}}\right.$, $\partial_{\vartheta}$ ) with an equivalent integral form as follows:

$$
\begin{aligned}
\mathcal{Q}_{b} & =\frac{\partial}{\partial \bar{\vartheta}}\left[i \dot{\bar{F}}^{(b)}(\tau, \bar{\vartheta}) F^{(b)}(\tau, \bar{\vartheta})-i \bar{F}^{(b)}(\tau, \bar{\vartheta}) \dot{F}^{(b)}(\tau, \bar{\vartheta})\right] \\
& \equiv \int d \bar{\vartheta}\left[i \dot{\bar{F}}^{(b)}(\tau, \bar{\vartheta}) F^{(b)}(\tau, \bar{\vartheta})-i \bar{F}^{(b)}(\tau, \bar{\vartheta}) \dot{F}^{(b)}(\tau, \bar{\vartheta})\right], \\
Q_{a b} & =\frac{\partial}{\partial \vartheta}\left[i \bar{F}^{(a b)}(\tau, \vartheta) \dot{F}^{(a b)}(\tau, \vartheta)-i \dot{\bar{F}}^{(a b)}(\tau, \vartheta) F^{(a b)}(\tau, \vartheta)\right] \\
& \equiv \int d \vartheta\left[i \bar{F}^{(a b)}(\tau, \vartheta) \dot{F}^{(a b)}(\tau, \vartheta)-i \dot{\bar{F}}^{(a b)}(\tau, \vartheta) F^{(a b)}(\tau, \vartheta)\right], \\
Q_{d} & =\frac{\partial}{\partial \vartheta}\left[i \bar{F}^{(d)}(\tau, \vartheta) \dot{F}^{(d)}(\tau, \vartheta)-i \dot{\bar{F}}^{(d)}(\tau, \vartheta) F^{(d)}(\tau, \vartheta)\right] \\
& \equiv \int d \vartheta\left[i \bar{F}^{(d)}(\tau, \vartheta) \dot{F}^{(d)}(\tau, \vartheta)-i \dot{\bar{F}}^{(d)}(\tau, \vartheta) F^{(d)}(\tau, \vartheta)\right], \\
\mathbb{Q}_{a d} & =\frac{\partial \bar{\vartheta}}{\partial \bar{\vartheta}}\left[i \dot{\bar{F}}^{(a d)}(\tau, \bar{\vartheta}) F^{(a d)}(\tau, \bar{\vartheta})-i \bar{F}^{(a d)}(\tau, \bar{\vartheta}) \dot{F}^{(a d)}(\tau, \bar{\vartheta})\right] \\
& \equiv \int d \bar{\vartheta}\left[i \dot{\bar{F}}^{(a d)}(\tau, \bar{\vartheta}) F^{(a d)}(\tau, \bar{\vartheta})-i \bar{F}^{(a d)}(\tau, \bar{\vartheta}) \dot{F}^{(a d)}(\tau, \bar{\vartheta})\right],
\end{aligned}
$$

where the superscripts $(b)$ and $(a b)$ stand for the antichiral and chiral supervariables that have been obtained after the application of the BRST and anti-BRST invariant restrictions, respectively. The superscripts $(d)$ and $(a d)$ show the chiral and antichiral supervariables that are obtained after the application of co-BRST and anti-co-BRST invariant restrictions, respectively. It is clear that the nilpotency $\left(\partial_{\bar{\vartheta}}^{2}=0, \partial_{\vartheta}^{2}=0\right)$ of the translational generators $\left(\partial_{\bar{\vartheta}}, \partial_{\vartheta}\right)$ implies that

$$
\begin{aligned}
& \partial_{\bar{\vartheta}} Q_{b}=0 \Leftrightarrow s_{b} Q_{b}=-i\left\{Q_{b}, Q_{b}\right\}=0, \\
& \partial_{\vartheta} \mathscr{Q}_{a b}=0 \Leftrightarrow s_{a b} \mathscr{Q}_{a b}=-i\left\{\mathscr{Q}_{a b}, \mathscr{Q}_{a b}\right\}=0 \text {, } \\
& \partial_{\vartheta} \mathscr{Q}_{d}=0 \Leftrightarrow s_{d} \mathscr{Q}_{d}=-i\left\{\mathscr{Q}_{d}, \mathscr{Q}_{d}\right\}=0, \\
& \partial_{\bar{\vartheta}} \mathscr{Q}_{a d}=0 \Leftrightarrow s_{a d} \mathscr{Q}_{a d}=-i\left\{\mathscr{Q}_{a d}, \mathbb{Q}_{a d}\right\}=0 \text {, }
\end{aligned}
$$

which show the nilpotency $\left[Q_{(a) b}^{2}=Q_{(a) d}^{2}=0\right]$ of the conserved charges within the ambit of ACSA to BRST formalism. Thus, we have shown that there is a deep connection between the nilpotency $\left(\partial_{\bar{\vartheta}}^{2}=0, \partial_{\mathcal{9}}^{2}=0\right)$ of the translational generator $\left(\partial_{\bar{\vartheta}}, \partial_{\vartheta}\right)$ and the nilpotency (i.e., $\left.\mathcal{Q}_{(a) b}^{2}=Q_{(a) d}^{2}=0\right)$ of the (anti-)BRST and (anti-)co-BRST charges $\left[\mathcal{Q}_{(a) b}, \mathcal{Q}_{(a) d}\right.$ ]. The above nilpotency property can be also captured in an ordinary space where we use the (anti-)BRST exact as well as (anti-)co-BRST exact forms of the charges, namely,

$$
\begin{gathered}
Q_{b}=-i s_{b}(\bar{C} \dot{C}-\dot{\bar{C}} C), \\
Q_{a b}=+i s_{a b}(\bar{C} \dot{C}-\dot{\bar{C}} C), \\
Q_{d}=i s_{d}(\bar{C} \dot{C}-\dot{\bar{C}} C), \\
Q_{a d}=-i s_{a d}(\bar{C} \dot{C}-\dot{\bar{C}} C) .
\end{gathered}
$$

The above expressions show the nilpotency property of the (anti-)BRST along with (anti-)co-BRST conserved charges, in a simpler way, in an ordinary space (cf. (74)).

Now, we are in a stage to show the absolute anticommutativity of the (anti-)BRST along with (anti-)co-BRST charges. For this purpose, we write the charges in terms of the (anti-)chiral supervariables and the derivatives $\left(\partial_{\vartheta}, \partial_{\bar{\vartheta}}\right)$ of the Grassmannian variables $(\bar{\vartheta}, \vartheta)$

$$
\begin{gathered}
Q_{b}=-i \frac{\partial}{\partial \vartheta}\left[\dot{F}^{(a b)}(\tau, \vartheta) F^{(a b)}(\tau, \vartheta)\right] \equiv-i \int d \vartheta\left[\dot{F}^{(a b)}(\tau, \vartheta) F^{(a b)}(\tau, \vartheta)\right], \\
\mathcal{Q}_{a b}=i \frac{\partial}{\partial \bar{\vartheta}}\left[\dot{\bar{F}}^{(b)}(\tau, \bar{\vartheta}) \bar{F}^{(b)}(\tau, \bar{\vartheta})\right] \equiv i \int d \bar{\vartheta}\left[\dot{\bar{F}}^{(b)}(\tau, \bar{\vartheta}) \bar{F}^{(b)}(\tau, \bar{\vartheta})\right] \\
\mathcal{Q}_{d}=i \frac{\partial}{\partial \bar{\vartheta}}\left[\dot{\bar{F}}^{(a d)}(\tau, \bar{\vartheta}) \bar{F}^{(a d)}(\tau, \bar{\vartheta})\right] \equiv i \int d \bar{\vartheta}\left[\dot{\bar{F}}^{(a d)}(\tau, \bar{\vartheta}) \bar{F}^{(a d)}(\tau, \bar{\vartheta})\right], \\
\mathcal{Q}_{a d}=-i \frac{\partial}{\partial \vartheta}\left[\dot{F}^{(d)}(\tau, \vartheta) F^{(d)}(\tau, \vartheta)\right] \equiv-i \int d \vartheta\left[\dot{F}^{(d)}(\tau, \vartheta) F^{(d)}(\tau, \vartheta)\right],
\end{gathered}
$$

where the superscripts $(a) b$ and $(a) d$ denote the same meaning as explained earlier. Here, it is straightforward to check that the nilpotency $\left(\partial_{\bar{\vartheta}}^{2}=0, \partial_{\vartheta}^{2}=0\right)$ of the translational generators $\left(\partial_{\bar{\vartheta}}, \partial_{\vartheta}\right)$ implies the following relations:

$$
\begin{aligned}
& \partial_{\vartheta} Q_{b}=0 \Leftrightarrow s_{a b} Q_{b}=-i\left\{Q_{b}, Q_{a b}\right\}=0, \\
& \partial_{\bar{\vartheta}} Q_{a b}=0 \Leftrightarrow s_{b} Q_{a b}=-i\left\{Q_{a b}, Q_{b}\right\}=0, \\
& \partial_{\bar{\vartheta}} Q_{d}=0 \Leftrightarrow s_{a d} Q_{d}=-i\left\{Q_{d}, Q_{a d}\right\}=0, \\
& \partial_{\vartheta} Q_{a d}=0 \Leftrightarrow s_{d} Q_{a d}=-i\left\{Q_{a d}, Q_{d}\right\}=0,
\end{aligned}
$$

which show the absolute anticommutativity property of the (anti-)BRST as well as (anti-)co-BRST conserved charges. The property of absolute anticommutativity of conserved charges can also be captured explicitly in an ordinary space by using the following (anti-)BRST exact and (anti-)coBRST exact forms of the charges, namely, 


$$
\begin{aligned}
& Q_{b}=-i s_{a b}(\dot{C} C), Q_{a b}=+i s_{b}(\dot{\bar{C}} \bar{C}), \\
& Q_{d}=i s_{a \mathrm{~d}}(\dot{\bar{C}} \bar{C}), Q_{a d}=-i s_{d}(\dot{C} C) .
\end{aligned}
$$

\section{Invariances of Lagrangian in ACSA}

In this section, we discuss the (anti-)BRST along with (anti)co-BRST invariances of the Lagrangian (7) within the scope of ACSA to BRST formalism. For this purpose, foremost, we generalize the ordinary Lagrangian of $(0+1)$-dimensional onto the suitably chosen $(1,1)$-dimensional (anti-)chiral super-submanifold of the most common $(1,2)$-dimensional supermanifold. The expressions of the (anti-)chiral super Lagrangian are

$$
\begin{aligned}
L(\tau) \longrightarrow \tilde{L}^{(a c)}(\tau, \bar{\vartheta})= & \dot{r}(\tau) p_{r}(\tau)+\dot{\Theta}^{(b)}(\tau, \bar{\vartheta}) p_{\varphi}(\tau) \\
& -\frac{1}{2} p_{r}^{2}(\tau)-\frac{1}{2 r^{2}} p_{\varphi}^{2}(\tau) \\
& -\mathscr{Z}^{(b)}(\tau, \bar{\vartheta}) p_{\varphi}(\tau)-V(r) \\
& +\frac{1}{2} \mathscr{B}^{2}(\tau)+\mathscr{B}(\tau) \\
& \cdot\left[\dot{\mathscr{Z}}^{(b)}(\tau, \bar{\vartheta})+\Theta^{(b)}(\tau, \bar{\vartheta})\right] \\
& -i \dot{\bar{F}}^{(b)}(\tau, \bar{\vartheta}) \dot{C}(\tau)+i \bar{F}^{(b)}(\tau, \bar{\vartheta}) C(\tau), \\
L(\tau) \longrightarrow \tilde{L}^{(c)}(\tau, \vartheta)= & \dot{r}(\tau) p_{r}(\tau)+\dot{\Theta}^{(a b)}(\tau, \vartheta) p_{\varphi}(\tau) \\
& -\frac{1}{2} p_{r}^{2}(\tau)-\frac{1}{2 r^{2}} p_{\varphi}^{2}(\tau) \\
& -\mathscr{Z}^{(a b)}(\tau, \vartheta) p_{\varphi}(\tau)-V(r) \\
& +\frac{1}{2} \mathscr{B}^{2}(\tau)+\mathscr{B}(\tau) \\
& \cdot\left[\dot{\mathscr{Z}}^{(a b)}(\tau, \vartheta)+\Theta^{(a b)}(\tau, \vartheta)\right] \\
& -i \dot{\bar{C}}^{(\tau)} \dot{F}^{(a b)}(\tau, \vartheta)+i \bar{C}(\tau) F^{(a b)}(\tau, \vartheta),
\end{aligned}
$$

where the superscripts $(a c)$ and $(c)$ on the super Lagrangians denote the antichiral and chiral super Lagrangians (containing antichiral and chiral supervariables), respectively. It is evident that under the application of translational generators $\left(\partial_{\bar{\vartheta}}, \partial_{\vartheta}\right)$, we get the (anti-)BRST invariance of Lagrangian $(L)$ with the following results:

$$
\begin{aligned}
\frac{\partial}{\partial \bar{\vartheta}}\left[\tilde{L}^{(a c)}(\tau, \bar{\vartheta})\right] & =\frac{d}{d \tau}[\mathscr{B}(\tau) \dot{C}(\tau)], \\
\frac{\partial}{\partial \vartheta}\left[\tilde{L}^{(c)}(\tau, \vartheta)\right] & =\frac{d}{d \tau}[\mathscr{B}(\tau) \dot{\bar{C}}(\tau)],
\end{aligned}
$$

which imply that the generalized version of super Lagrangians remains quasi-invariant (i.e., up to a total time derivative) under the translational generators $\left(\partial_{\bar{\vartheta}}, \partial_{\vartheta}\right)$ within the scope of ACSA which are consistent with Equation (10).
Now, we capture the (anti-)co-BRST invariance of the Lagrangian (7) within the scope of (anti-)chiral supervariable approach. For this, we generalize the ordinary Lagrangian into (anti-)co-BRST super Lagrangian where $(0+1)$ -dimensional theory is generalized onto the $(1,1)$-dimensional (anti-)chiral super-submanifold of the common (1,2 )-dimensional supermanifold as follows:

$$
\begin{aligned}
L(\tau) \longrightarrow \tilde{L}^{(c, d)}(\tau, \vartheta)= & \dot{r}(\tau) p_{r}(\tau)+\dot{\Theta}^{(d)}(\tau, \vartheta) p_{\varphi}(\tau) \\
& -\frac{1}{2} p_{r}^{2}(\tau)-\frac{1}{2 r^{2}} p_{\varphi}^{2}(\tau) \\
& -\mathscr{Z}^{(d)}(\tau, \vartheta) p_{\varphi}(\tau)-V(r) \\
& +\frac{1}{2} \mathscr{B}^{2}(\tau)+\mathscr{B}(\tau) \\
& \cdot\left[\dot{\mathscr{Z}}^{(d)}(\tau, \vartheta)+\Theta^{(d)}(\tau, \vartheta)\right] \\
& -i \dot{\bar{C}}(\tau) \dot{F}^{(d)}(\tau, \vartheta)+i \bar{C}(\tau) F^{(d)}(\tau, \vartheta), \\
L(\tau) \longrightarrow \tilde{L}^{(a c, a d)}(\tau, \bar{\vartheta})= & \dot{r}(\tau) p_{r}(\tau)+\dot{\Theta}^{(a d)}(\tau, \bar{\vartheta}) p_{\varphi}(\tau) \\
& -\frac{1}{2} p_{r}^{2}(\tau)-\frac{1}{2 r^{2}} p_{\varphi}^{2}(\tau) \\
& -\mathscr{Z}^{(a d)}(\tau, \bar{\vartheta}) p_{\varphi}(\tau)-V(r) \\
& +\frac{1}{2} \mathscr{B}^{2}(\tau)+\mathscr{B}(\tau) \\
& \cdot\left[\dot{\mathscr{Z}}^{(a d)}(\tau, \bar{\vartheta})+\Theta^{(a d)}(\tau, \bar{\vartheta})\right] \\
& -i \dot{\bar{F}}^{(a d)}(\tau, \bar{\vartheta}) \dot{C}(\tau)+i \bar{F}^{(a d)}(\tau, \bar{\vartheta}) C(\tau),
\end{aligned}
$$

where the superscripts $(c, d)$ and $(a c, a d)$ denote the super Lagrangians (containing the chiral and antichiral supervariables) obtained after the application of the coBRST and anti-co-BRST invariant restrictions, respectively. It is straightforward to check that

$$
\begin{gathered}
\frac{\partial}{\partial \vartheta}\left[\tilde{L}^{(c, d)}(\tau, \vartheta)\right]=-\frac{d}{d \tau}\left[p_{\varphi}(\tau) \dot{\bar{C}}(\tau)\right], \\
\frac{\partial}{\partial \bar{\vartheta}}\left[\tilde{L}^{(a c, a d)}(\tau, \bar{\vartheta})\right]=-\frac{d}{d \tau}\left[p_{\varphi}(\tau) \dot{C}(\tau)\right],
\end{gathered}
$$

which show the (anti-)co-BRST invariance of the Lagrangian $L$ within the ambit of ACSA to BRST formalism. At the end of this section, we have the following concluding remarks. There are deep connections between the (anti)BRST symmetries $\left(s_{(a) b}\right)$ and derivatives $\left(\partial_{\bar{\vartheta}}, \partial_{\vartheta}\right)$ of the Grassmannian variables $(\bar{\vartheta}, \vartheta)$ with the following mappings: $s_{b} \leftrightarrow \partial_{\bar{\vartheta}}$ and $s_{a b} \leftrightarrow \partial_{\vartheta}$. Similarly, in the case of (anti-)coBRST symmetry transformations, it is clear that these symmetry transformations are also connected with the derivatives $\left(\partial_{\bar{\vartheta}}, \partial_{\vartheta}\right)$ of Grassmannian variables with the mappings: $s_{d} \leftrightarrow \partial_{\vartheta}$ and $s_{a d} \leftrightarrow \partial_{\bar{\vartheta}}$ (cf. Sections 4 and 5). 


\section{Conclusions}

In our present analysis, for the first time, we have derived the off-shell nilpotent quantum (anti-)BRST along with (anti)co-BRST symmetry transformations within the scope of ACSA. We have also discussed the nilpotency along with absolute anticommutativity properties of the corresponding (anti-)BRST along with (anti-)co-BRST conserved charges of the ordinary $(0+1)$-dimensional gauge invariant Christ-Lee model within the ambit of (anti-)chiral supervariable approach (ACSA) to BRST formalism.

The novel remarks of our present endeavor are the derivation of the off-shell nilpotent (anti-)BRST along with (anti)co-BRST symmetry transformations (cf. Section 4) and the proof of nilpotency and the anticommutativity properties of the (anti-)BRST and (anti-)co-BRST charges in spite of the fact that we have taken into account only the (anti-)chiral super expansions of the supervariables (cf. Section 5). The nilpotency and anticommutativity properties of the above conserved charges and derivation of the corresponding (anti)BRST and (anti-)co-BRST symmetry transformations are obvious when the full super expansions of the supervariables (i.e., BT-supervariable formalism [29-32]) are taken into account. However, for the present study, we have shown these properties with the help of only (anti-)chiral super expansions of the (anti-)chiral supervariables.

It is worthwhile to mention that the nilpotency of the BRST as well as anti-BRST conserved charges is connected with the nilpotency $\left(\partial_{\overline{9}}^{2}=\partial_{9}^{2}=0\right)$ of the translational generators $\partial_{\bar{\vartheta}}$ and $\partial_{\vartheta}$, respectively. On the other hand, nilpotency of the co-BRST and anti-co-BRST charges is connected with nilpotency $\left(\partial_{\vartheta}^{2}=\partial_{\overline{9}}^{2}=0\right)$ of the translational generators $\partial_{\vartheta}$ and $\partial_{\bar{\vartheta}}$, respectively. However, we have shown (cf. Section 5) that the absolute anticommutativity of the BRST charge with anti-BRST charge is connected with the nilpotency $\left(\partial_{\vartheta}^{2}=0\right)$ of the translational generator $\left(\partial_{\vartheta}\right)$ and absolute anticommutativity of anti-BRST charge with BRST charge is connected with the nilpotency $\left(\partial_{\overline{9}}^{2}=0\right)$ of the translational generator $\left(\partial_{\overline{9}}\right)$. On the contrary, the absolute anticommutativity of the co-BRST charge with anti-co-BRST charge is connected with the nilpotency of the translational generator $\left(\partial_{\bar{\vartheta}}\right)$ and the absolute anticommutativity of the anti-co-BRST charge with co-BRST charge is deeply related with the nilpotency of the translational generator $\left(\partial_{\vartheta}\right)$. These statements are completely novel for the present model. We have also captured the (anti-)BRST along with (anti-)co-BRST invariances of the Lagrangian within the scope of ACSA. In fact, the action corresponding to the (anti-)chiral super Lagrangian is independent of Grassmannian variables $(\vartheta, \bar{\vartheta})$ which is completely novel for the present CL model (cf. Section 6).

The above issues, within the scope of ACSA to BRST approach, would be discussed in our future investigations for the various gauge-invariant models/theories like ABJM theory [33-35], supersymmetric Chern-Simons theory [36], Jackiw-Pi model, Freedman-Townsend model, and Abelian gauge theory with higher derivative matter fields. In fact, our standard techniques of ACSA to BRST formalism are applicable wherever gauge invariance is present in the the- ory. Furthermore, there is an interesting and important work [37] that would be discussed in the future for different prospects of the theoretical and physical point of view in the domain of theoretical high-energy physics.

\section{Data Availability}

No data were used to support this study.

\section{Additional Points}

arXiv identifier. arXiv: 2102.03845 [hep-th]. A version of this work is available as a preprint at [38].

\section{Conflicts of Interest}

The authors of this study declare that there are no conflicts of interest.

\section{Acknowledgments}

B. Chauhan and S. Kumar are thankful to the DST-INSPIRE and BHU fellowships for financial support, respectively. The authors also thank Dr. R. Kumar for a careful reading of the manuscript and for important as well as significant suggestions.

\section{References}

[1] P. A. M. Dirac, Lectures on Quantum Mechanics, Belfer Graduate School of Science, Yeshiva University Press, New York, 1964.

[2] K. Sundermeyer, Constrained Dynamics: Lecture Notes in Physics, vol. 169, Springer-Verlag, Berlin, 1982.

[3] C. Becchi, A. Rouet, and R. Stora, "The abelian Higgs Kibble model, unitarity of the S-operator," Physics Letters B, vol. 52, no. 3, pp. 344-346, 1974.

[4] C. Becchi, A. Rouet, and R. Stora, "Renormalization of the abelian Higgs-Kibble model," Communications in Mathematical Physics, vol. 42, no. 2, pp. 127-162, 1975.

[5] C. Becchi, A. Rouet, and R. Stora, "Renormalization of gauge theories," Annals of Physics, vol. 98, no. 2, pp. 287-321, 1976.

[6] I. V. Tyutin, "Lebedev institute preprint, report number: FIAN-39," 1975, https://arxiv.org/abs/0812.0580.

[7] S. Gupta and R. P. Malik, "Rigid rotor as a toy model for Hodge theory," European Physical Journal C: Particles and Fields, vol. 68, no. 1-2, pp. 325-335, 2010.

[8] D. Shukla, T. Bhanja, and R. P. Malik, "Supervariable approach to the nilpotent symmetries for a toy model of the Hodge theory," Advances in High Energy Physics, vol. 2016, Article ID 2618150, 13 pages, 2016.

[9] R. Kumar and A. Shukla, "Novel symmetries in Christ-Lee model," EPL (Europhysics Letters), vol. 115, no. 2, article 21003, 2016.

[10] R. Kumar and A. Shukla, "Christ-Lee model: augmented supervariable approach," Advances in High Energy Physics, vol. 2018, Article ID 7381387, 12 pages, 2018.

[11] L. Bonora and M. Tonin, "Superfield formulation of extended BRS symmetry," Physics Letters B, vol. 98, no. 1-2, pp. 48-50, 1981. 
[12] L. Bonora, P. Pasti, and M. Tonin, "Geometric description of extended BRS symmetry in superfield formulation," Nuovo Cimento A, vol. 63, no. 3, pp. 353-364, 1981.

[13] R. Delbourgo and P. D. Jarvis, "Extended BRS invariance and OSp (4/2) supersymmetry," Journal of Physics A: Mathematical and General, vol. 15, no. 2, pp. 611-625, 1982.

[14] N. H. Christ and T. D. Lee, "Operator ordering and Feynman rules in gauge theories," Physical Review D, vol. 22, no. 4, pp. 939-958, 1980.

[15] M. E. V. Costa and H. O. Girotti, "Quantization of gaugeinvariant theories through the Dirac-bracket formalism," Physical Review D, vol. 24, no. 12, pp. 3323-3325, 1981.

[16] M. E. V. Costa, H. O. Girotti, and T. J. M. Simoes, "Dynamics of gauge systems and Dirac's conjecture," Physical Review D, vol. 32, no. 2, pp. 405-410, 1985.

[17] S. K. Blau, "The Feynman path integral for constrained systems," Annals of Physics, vol. 205, no. 2, pp. 392-417, 1991.

[18] E. M. Rabei, "Quantization of Christ-Lee model using the WKB approximation," International Journal of Theoretical Physics, vol. 42, no. 9, pp. 2097-2102, 2003.

[19] U. Kulsheeshtha, D. S. Kulshreshtha, and H. J. W. MulerKirsten, "BRST formulation of the Christ-Lee model," Canadian Journal of Physics, vol. 73, no. 5-6, pp. 386-392, 1995.

[20] S. Anjali and S. Gupta, "Faddeev-Jackiw quantization of Christ-Lee model," International Journal of Modern Physics A: Particles and Fields; Gravitation; Cosmology; Nuclear Physics, vol. 35, no. 10, article 2050072, 2020.

[21] S. Kumar, B. Chauhan, and R. P. Malik, "(Anti-)chiral supervariable approach to nilpotent and absolutely anticommuting conserved charges of reparametrization invariant theories: a couple of relativistic toy models as examples," International Journal of Modern Physics A: Particles and Fields; Gravitation; Cosmology; Nuclear Physics, vol. 33, no. 22, article 1850133, 2018.

[22] B. Chauhan, S. Kumar, A. Tripathi, and R. P. Malik, "Modified 2D Proca theory: revisited under BRST and (anti-)chiral superfield formalisms," Advances in High Energy Physics, vol. 2020, Article ID 3495168, 38 pages, 2020.

[23] B. Chauhan, S. Kumar, and R. P. Malik, "Nilpotent charges in an interacting gauge theory and an $\mathcal{N}=2$ SUSY quantum mechanical model: (anti-)chiral superfield approach," International Journal of Modern Physics A: Particles and Fields; Gravitation; Cosmology; Nuclear Physics, vol. 34, no. 24, article 1950131, 2019.

[24] B. Chauhan, S. Kumar, and R. P. Malik, "(Anti-)chiral superfield approach to interacting Abelian 1-form gauge theories: nilpotent and absolutely anticommuting charges," International Journal of Modern Physics A: Particles and Fields; Gravitation; Cosmology; Nuclear Physics, vol. 33, no. 4, article 1850026, 2018.

[25] N. Srinivas, T. Bhanja, and R. P. Malik, "(Anti)chiral superfield approach to nilpotent symmetries: self-dual chiral bosonic theory," Advances in High Energy Physics, vol. 2017, Article ID 6138263, 14 pages, 2017.

[26] S. Krishna and R. P. Malik, "N=2SUSY symmetries for a moving charged particle under influence of a magnetic field: supervariable approach," Annals of Physics, vol. 355, pp. 204-216, 2015.

[27] S. Krishna and R. P. Malik, "A free $\$ \backslash$ mathcal $\{\mathrm{N}\}=2 \$$ supersymmetric system: novel symmetries," Europhysics Letters, vol. 109, no. 3, article 31001, 2015.
[28] S. Krishna, D. Shukla, and R. P. Malik, "Novel symmetries in an interacting $\mathcal{N}=2$ supersymmetric quantum mechanical model," International Journal of Modern Physics A: Particles and Fields; Gravitation; Cosmology; Nuclear Physics, vol. 31, no. 19 , article $1650113,2016$.

[29] R. P. Malik, "Superfield approach to symmetries for matter fields in Abelian gauge theories," Journal of Physics A: Mathematical and General, vol. 37, no. 19, pp. 5261-5274, 2004.

[30] R. P. Malik, "Superfield approach to nilpotent symmetries for QED from a single restriction: an alternative to the horizontality condition," Journal of physics a: mathematical and theoretical, vol. 39, no. 33, pp. 10575-10587, 2006.

[31] R. P. Malik, "An alternative to the horizontality condition in the superfield approach to BRST symmetries," The European Physical Journal C, vol. 51, no. 1, pp. 169-177, 2007.

[32] R. P. Malik, "Abelian 2-form gauge theory: superfield formalism," The European Physical Journal C, vol. 60, no. 3, pp. 457-470, 2009.

[33] M. Faizal and S. Upadhyay, "Spontaneous breaking of the BRST symmetry in the ABJM theory," Physics Letters B, vol. 736, pp. 288-292, 2014.

[34] F. Mir, S. Upadhyay, and B. P. Mandal, "Finite field-dependent BRST symmetry for ABJM theory in $\mathrm{N}=1$ superspace," Physics Letters B, vol. 738, pp. 201-205, 2014.

[35] S. Upadhyay, M. Faisal, and P. A. Ganai, "Interpolating between different gauges in the ABJM theory," International Journal of Modern Physics A: Particles and Fields; Gravitation; Cosmology; Nuclear Physics, vol. 30, no. 27, article 1550185, 2015.

[36] K. Hasebe, "Supersymmetric Chern-Simons theory and supersymmetric quantum Hall liquid," Physical Review D, vol. 74, no. 4, article 045026, 2006.

[37] M. Pavsic, "Classification of static plane symmetric spacetime via Noether gauge symmetries," International Journal of Geometric Methods in Modern Physics, vol. 13, no. 9, article 1650111, 2016.

[38] B. Chauhan and S. Kumar, "Christ-Lee model: (anti-)chiral supervariable approach to BRST formalism," 2021, https:// arxiv.org/abs/2102.03845. 